\title{
Thermodynamic characterization of a metallomesogenic stationary phase in gas chromatography
}

\author{
Jian-Lian Chen ${ }^{\mathrm{a}, *}$, Chuen-Ying Liu ${ }^{\mathrm{b}}$ \\ ${ }^{a}$ Department of Occupational Safety and Health, Chung Shan Medical University, Taichung 40201, Taiwan \\ ${ }^{\mathrm{b}}$ Department of Chemistry, National Taiwan University, Taipei, Taiwan
}

Received 5 May 2005; received in revised form 30 May 2005; accepted 31 May 2005

Available online 14 July 2005

\begin{abstract}
The nine representative dialkylsulfides are designed as probes to the assessment of the discotic copper complex-containing siloxane polymer as the GC stationary phase and the probable mechanism implicated. According to the isothermal retention data, which are transformed to the enthalpy, entropy and Gibbs energy losses as the gaseous probes partitioning in a mesophase and between mesophases, first we roughly observe the solutes with branched alkyl substituents were profoundly attracted to discotic lamellar phase, those with electron-releasing substituents to lamellar crystalline phase, and those with disk-like substituents to discotic hexagonal phase. To quantify the correspondent interactions between gaseous solutes and these mesophases, three controlling descriptors are chosen to account for the general dispersion, Lewis acid-base, and polarizability from unpaired-electrons within interactions. Through the multi-linear regression between enthalpy losses and solute descriptors, four linear equations, which correlation coefficients are larger than 0.926 , are given to show the responsible extent that the possible interactions would be involved in and are agreeably consistent with the rough observation. The schematic representation of these mesomorphic structures clearly elucidates the connection between the proposed mechanisms and direct observations.
\end{abstract}

(C) 2005 Elsevier B.V. All rights reserved.

Keywords: Dialkylsulfide; Gas chromatography; Stationary phase; Thermodynamic; Metallomesogen; Multi-linear regression

\section{Introduction}

Metallomesogens exploit the opulent possibility of combining the properties of liquid crystals (fluidity, ease of processability, one- or two-dimensional order, etc.) with the properties associated with metal atoms (color, paramagnetism, an electron-rich environment, etc.) [1]. Compounds showing interesting magnetic [2], electrical [3], optical [4], and electrooptical [5] properties have already been obtained. However, we focus our interest to the analytical application of the GC stationary phases. A series of nickel-, zinc-thiolates or copper-carboxylates complexes showing calamitic or discotic mesophases were designed as the coating materials on the Chromosorb W for packing columns [6-8] and as the

\footnotetext{
* Corresponding author. Tel.: +886 4 24730022; fax: +886 423248194.

E-mail address: cj1@csmu.edu.tw (J.-L. Chen).
}

active moieties introducing into the siloxane polymeric backbone for wall-coated capillaries [8-11]. In general, the specified thermal behavior of these mesomorphic phases exhibited not only the separation of geometric isomers due to the benefits of the orientated ordering of liquid crystal molecules, but also electron-donating analytes due to the ligand exchange interactions committed to the coordination centers of metal ions.

Effective separations require thermodynamic consideration of selectivity optimization achieved by either temperature variation or by using different stationary phases. The thermotropic metallomesogen could simultaneously provide several different stationary phases as temperature changes. The selectivity of a stationary phase is defined as its relative contribution to comprise specific intermolecular interactions such as dispersion, induction, orientation, hydrogen-bond formation, and charge-transfer complexation. Historically, 
the most important classification of GC stationary phases was the Rohrschneider-McReynolds system. The main difficulty of selectivity ranking of stationary phases based on retention index differences came from the selected solutes rarely representing a simply individual interaction $[12,13]$. Kollie and Poole reasoned that the size dependence of the solutes could be removed by separating the free energy into a cavity term and an interaction term, the latter being independent of solute size and representative of polar solute-solvent interaction [14]. While the enthalpy changes were also responsible for the correlations between the interactions, the sorbent coefficients were likewise calculated by the multiple linear regression analysis [15]. The authors have demonstrated that the Lewis acid-base chemistry on clay surfaces explains the significant difference in enthalpy magnitudes between the sulfide and thiol classes.

In this study, dialkylsulfides, which contain a sulfur atom with donating electrons and two alkyl substituents with various dimensions and electron-releasing ability, are employed as probes to investigate the possible intermolecular interactions, with which the synthesized discotic copper-carboxylates mesophases would be concerned. The preferable selectivity toward respective probes in certain mesophases was assessed by the thermodynamic parameters obtained. Furthermore, we propose a multi-linear model, which could quantitatively reflect the potent influence of the sorbent morphology on the partition interactions.

\section{Experimental}

\subsection{Apparatus}

The gas chromatograph used throughout for column evaluation was a Shimadzu model 17A, equipped with a capillary column split-injection system and a flame photometric detector (FPD) detector. The major components of FPD were a $394 \mathrm{~nm}$ filter, a base block and a head block. To prevent the condensation of samples within the blocks, the temperature setting of base block should be 30 degrees higher than the oven temperature and the setting for the head block was $150^{\circ} \mathrm{C}$. High purity nitrogen was used as the carrier gas. Chromatograms were plotted on a Shimadzu CR-6A Chromatopac integrator.

\subsection{Materials}

Nine dialkylsulfides were obtained both from the Aldrich (USA) and TCI (Tokyo, Japan) and are listed in Table 1 with some physical and chemical properties. The stock solutions for these analytes were prepared $4 \times 10^{3} \mathrm{ppm}$ in $n$-hexane and kept at $4{ }^{\circ} \mathrm{C}$.

Deactivated fused silica capillaries $(20 \mathrm{~m} \times 0.25 \mathrm{~mm}$ i.d., Restek, Bellefonte, PA) were cleaned to remove impurities on the capillary wall surface prior to the dynamic coating of $\mathrm{P}_{-} \mathrm{C}_{15} \mathrm{CuC}_{18}$, a discotic copper complex-containing silox-
Table 1

Physical and chemical properties of dialkylsulfides ${ }^{\mathrm{a}}$

\begin{tabular}{lrlll}
\hline Sulfides & bp & $\log L^{16 \mathrm{~b}}$ & $\sum \beta_{2}^{\mathrm{Hb}}$ & $E^{\mathrm{b}}$ \\
\hline Group I & & & & \\
$\quad t$-Butyl methylsulfide & 102 & 2.985 & 0.298 & 0.101 \\
$\quad$ Diethylsulfide & 92 & 2.898 & 0.182 & 0.122 \\
Di- $i$ so-propylsulfide & 121 & 3.540 & 0.218 & 0.092 \\
Di- $t$-butylsulfide & 147 & 4.070 & 0.284 & 0.080 \\
Diallylsulfide & 138 & 3.682 & 0.228 & 0.376 \\
& & & & \\
Group II & 142 & 3.896 & 0.182 & 0.122 \\
$\quad$ Di- $n$-propylsulfide & 165 & 4.538 & 0.218 & 0.092 \\
Di-sec-butylsulfide & 142 & 3.753 & 0.168 & 0.330 \\
Pentamethylene sulfide & 188 & 4.894 & 0.182 & 0.122 \\
Di- $n$-butylsulfide & &
\end{tabular}

${ }^{a}$ bp: boiling point $\left({ }^{\circ} \mathrm{C}\right) ; \sum \beta_{2}^{\mathrm{H}}$ : solute hydrogen-bond basicity values; $\log L^{16}$ : solute gas-hexadecane partition coefficient; $E$ : the excess molar refraction.

b Calculated from [15].

ane polymer. All chemicals and the method for the synthesis and the capillary wall coating of $\mathrm{P}_{-} \mathrm{C}_{15} \mathrm{CuC}_{18}$ were clear described in [10].

\subsection{Measurement conditions}

To approximate zero surface coverage, a split/splitless injector was set at 30:1 split ratio and $1 \mu \mathrm{L}$ solutions of diluted concentration, $5 \mathrm{ppm}$ for Group I analytes and $2 \mathrm{ppm}$ for Group II in Table 1, were injected to the GC-FPD. The retention time of each solute was measured five times at 10$\mathrm{kPa}$ inlet pressure, which impelled the capillary flow rate toward $0.1 \mathrm{~cm} / \mathrm{s}$, and at the oven temperature between 30 and $95^{\circ} \mathrm{C}$ with an interval of $5^{\circ} \mathrm{C}$. The injector chamber was held at $250^{\circ} \mathrm{C}$. The gas-hold-up time was determined by the injection of $n$-hexane to the GC-FID system.

\section{Results and discussion}

Gas chromatography is often the method of choice for the determination of reliable thermodynamic data for volatile solutes, under conditions of approximately infinite dilution. The thermodynamic properties obtained can provide information on the factors that are important in solute-liquid crystalline molecular interactions and how they correspond to the mesomorphic textures. This information is ultimately useful for the discrimination of selectivity and further developments of the logical design of a particular stationary mesophase for a desired separation. In fact, a commercial capillary has already become available in 1991 lined with a film of mesogenic polymeric methylsiloxane [16].

\subsection{Thermodynamic behavior}

Although Görgényi submitted a newer approach to the estimation of molar heat capacities [17], the infinite-dilution solute partial molar enthalpy $(\Delta H)$ and entropy $(\Delta S)$ of solu- 
tion can both be obtained by means of the following equations [18-20]:

$\ln V_{\mathrm{g}}^{\mathrm{o}}=\frac{-\Delta H}{R T}+\frac{\Delta S}{R}-\ln \left(\frac{M}{273.3 R}\right)$

$V_{\mathrm{g}}^{\mathrm{o}}=\frac{V^{\prime}}{W}$

$V^{\prime}=\left(t_{\mathrm{r}}-t_{\mathrm{o}}\right) \times f$

where $t_{\mathrm{r}}$ is the retention time of the sample; $t_{\mathrm{o}}$ the retention time of the non-adsorbed substance; $V^{\prime}$ the retention volume; $f$ the flow rate of the carrier gas; $W$ the weight of the stationary phase; $M$ the molecular weight of the stationary phase; $R$ gas constant, $8.314\left(\mathrm{~J} \mathrm{~K}^{-1} \mathrm{~mol}^{-1}\right)$; and $V_{\mathrm{g}}^{\mathrm{o}}$ the specific retention volume.

By combining the equations shown above, $\Delta H$ can be calculated from the slope of the $\ln \left(t_{\mathrm{r}}-t_{\mathrm{o}}\right)$ (min) versus $T^{-1}$ (Kelvin temperature) plot. The results with standard deviations are summarized in Table 2 and they show that the little uncertainties were not influential. The sequence arrangement of nine dialkylsulfides in the first column in Table 2 was according to the elution order, which could apparently be divided into two groups. The setting of GC oven temperature, which governed the conformation of stationary phase, would be adjusted to properly retain vaporized solutes with various pressures, which sources occur in the injector chamber $\left(250^{\circ} \mathrm{C}\right)$. Hence, the oven temperature range, $35-70^{\circ} \mathrm{C}$, was assigned for Group I analytes and $60-95{ }^{\circ} \mathrm{C}$ for Group II. The stationary phases upon the $\mathrm{P}-\mathrm{C}_{15} \mathrm{CuC}_{18}$ modified capillary will exhibit the discotic lamellar $\left(\mathrm{D}_{\mathrm{L}}\right)$ phase and the lamellar crystalline phase $(\mathrm{K})$ in the former temperature range and discotic hexagonal phase $\left(\mathrm{D}_{\mathrm{ho}}\right)$ and $\mathrm{D}_{\mathrm{L}}$ phase in the latter temperature range [10].

To easily clarify the separation mechanism of the solutes in a given metallomesogen, differences in the respective thermodynamic parameters need to be considered. The results in Table 2 would indicate the enthalpy and entropy differences of solutes between two mesophases. For the assessment of Group I solutes, $\Delta H_{(\mathrm{K}-\mathrm{L})}$ assigned as $\Delta H_{(\mathrm{g}-\mathrm{L})}-\Delta H_{(\mathrm{g}-\mathrm{K})}$ is in the decreasing order of $t$-butyl methylsulfide $>$ diethylsulfide $>$ di-iso-propylsulfide $>$ diallylsulfide $>$ di- $t$-butylsulfide. The $\Delta S_{(\mathrm{K}-\mathrm{L})}$ order is same as $\Delta H_{(\mathrm{K}-\mathrm{L})}$. The greater $\Delta H_{(\mathrm{K}-\mathrm{L})}$ would experience stronger solute-K phase interaction. In a similar manner, the greater $\Delta S_{(\mathrm{K}-\mathrm{L})}$ indicates that the solutes are more compatible with the $\mathrm{K}$ phase than with the $\mathrm{D}_{\mathrm{L}}$ phase. With the highest $\log L^{16}$ and negative values of $\Delta H_{(\mathrm{K}-\mathrm{L})}$ and $\Delta S_{(\mathrm{K}-\mathrm{L})}$, di- $t$-butylsulfide is exceptional among Group I solutes. This special tendency to dissolve in $\mathrm{D}_{\mathrm{L}}$ phase may be owed to the exoergic dispersion force exerted by branched alkyl substituents. Further we calculate the Gibbs free energy $(\Delta G)$ in Table 2 at the coexistent temperature of $\mathrm{K}$ and $\mathrm{D}_{\mathrm{L}}$ phase $\left(42.5^{\circ} \mathrm{C}\right)$. All are approaching zero, except $t$-butyl methylsulfide. This may be a result of basicity, which brings $t$-butyl

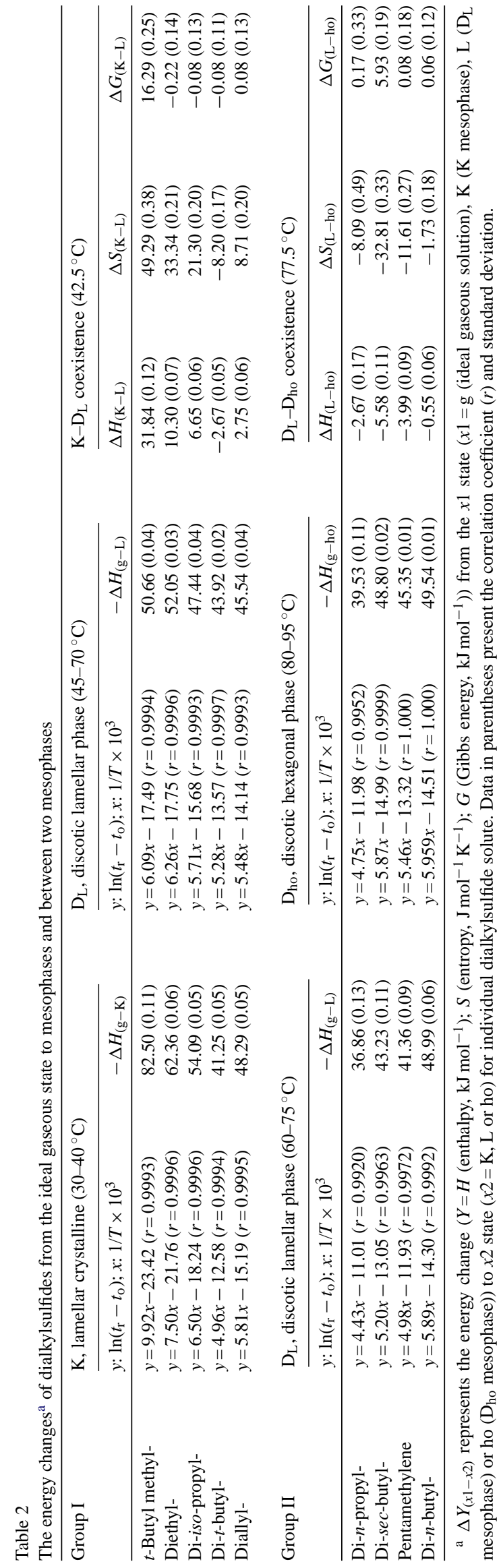


methylsulfide, bearing the highest $\beta_{2}^{\mathrm{H}}$, the more compatibility with the $\mathrm{K}$ phase than all other alkylsulfides in Group I.

As regards of Group II, negative $\Delta H_{(\mathrm{L}-\mathrm{ho})}$ and $\Delta S_{(\mathrm{L}-\mathrm{ho})}$ infer the preferable affinity for the discotic hexagonal $\left(D_{\text {ho }}\right)$ phase. The affinity order is in the decreasing order of di-sec-butylsulfide > pentamethylene sulfide > di- $n$ propylsulfide $>$ di- $n$-butylsulfide. This seems that the more disk-like the shape of molecular configuration is, the easier the molecule dissolves in $\mathrm{D}_{\text {ho }}$ phase. The calculated values of the Gibbs free energy in Table 2 at the coexistent temperature of $\mathrm{D}_{\mathrm{L}}$ and $\mathrm{D}_{\text {ho }}$ phase $\left(77.5^{\circ} \mathrm{C}\right)$ are near zero, except di-secbutylsulfide. This is originated from the largest entropy loss $\left(-32.81 \mathrm{~J} \mathrm{~mol}^{-1} \mathrm{~K}^{-1}\right)$ when the strong entrapment of di-secbutylsulfide from $\mathrm{D}_{\mathrm{L}}$ into $\mathrm{D}_{\text {ho }}$ occurred.

The mechanisms of retention could also be studied via the temperature dependence of the relative retention, $\alpha$ of isomeric solute pairs [18]. The relevant equation for the solute pair is as the following equation:

$\ln \alpha_{(\mathrm{B} / \mathrm{A})}=\frac{\Delta \Delta H_{\mathrm{B}-\mathrm{A}}}{R T}-\frac{\Delta \Delta S_{\mathrm{B}-\mathrm{A}}}{R}$

where $\Delta \Delta Y_{\mathrm{B}-\mathrm{A}}(Y=H$ or $S)$ indicates the $\Delta Y$ value for $\mathrm{B}$ minus that for A. $\Delta \Delta H_{\mathrm{B}-\mathrm{A}}$ and $\Delta \Delta S_{\mathrm{B}-\mathrm{A}}$ values with little standard deviation could be obtained from the slopes and intercepts of plots of $\ln \alpha$ versus $T^{-1}$ and are arranged in Table 3. With the comparison of the data in Group II, the negative values of Gibbs free energy reveal that the partition between Group I solutes and mesophases is thermodynamically very consistent with the gas-liquid chromatographic mechanism. Slightly positive $\Delta \Delta G_{\mathrm{B}-\mathrm{A}}$ values for Group II sulfides may imply the kinetic interference is somewhat involved.

\subsection{Multi-linear model}

More reliable descriptions to clarify the sources of solvency are needed, though the trend of dialkylsulfide probes distributed in the prepared mesophases has been generally described in previous section. Here, we correlated the measured enthalpy data with $\log L^{16}, \sum \beta_{2}^{\mathrm{H}}$ and/or $E$ descriptors upon the multi-linear regression (MLR), which may be determined by:

$-\Delta H_{(\mathrm{g}-\mathrm{X})}=c+e E+b \sum \beta_{2}^{\mathrm{H}}+l \log L^{16}$

where the subscript $\mathrm{X}$ represents variant mesophases and $c$, $e, b, l$ are coefficients (system constants) from the regression. These descriptors are suggested from the LSER model $[21,22] . \log L^{16}$, the gas-liquid partition coefficient on hexadecane at $25^{\circ} \mathrm{C}$, represents the contribution of cavity formation and general dispersion interactions in gas-condensed systems $[23,24]$. Wide variety of solute properties has been estimated including retention in gas chromatography, solubility in liquids, adsorption at solid and liquid interfaces, and gas-substrate distribution constants in biological and environmental systems [25,26]. Sulfur compounds are known

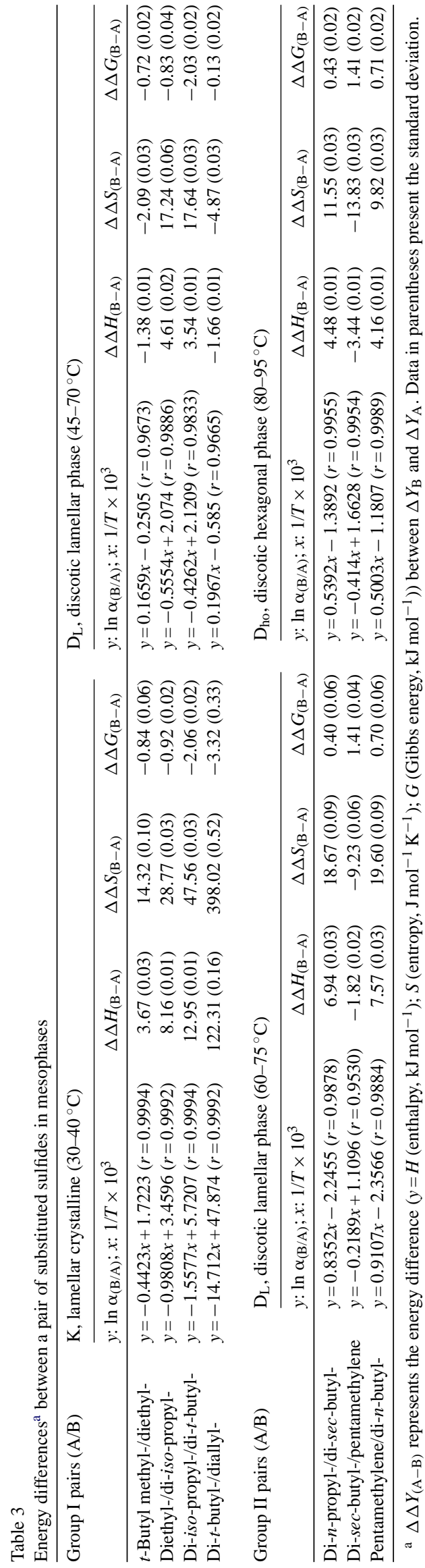


to behave as Lewis base. Therefore, solute hydrogen-bond basicity, $\sum \beta_{2}^{\mathrm{H}}$, values are needed to quantify the basicity of each of the dialkylsulfides. The solute excess molar refraction, $E$, models the polarizability contributions from $n$ - and $\pi$-electrons. That is the difference between the molar refractivity of a solute and the molar refractivity of an alkane with the same volume. Additional solute descriptors that are excluded here are the solute hydrogen-bond acidity, $\sum \alpha_{2}^{\mathrm{H}}$, and the solute dipolarity/polarizability, $\pi_{2}^{\mathrm{H}}$, because they are either statistically identical $\left(\pi_{2}^{\mathrm{H}}\right)$ or zero $\left(\sum \alpha_{2}^{\mathrm{H}}\right)$. As the comparison of system constants for different mesophases, these two descriptors will make no difference and contribute to the same effect on the constant term, $c$, in the above expression.

We try to search for the descriptor values of dialkylsulfides from starting with the review paper [24]. However, only information about diethylsulfide, di- $n$-propylsulfide and di- $n$-butylsulfide were obtained [27]. Under the overall consideration of the contribution of alkyl groups in sulfides, the descriptor's data in Table 1 are calculated from the additive fragment constants. The additivity of one set of 81 functional group fragments was capable of reproducing experimentally derived results with correlation coefficients ranging from 0.95 to 0.99 [28]. The important messages brought from substituted groups are surely considered and preserved by the group contribution approach.

The enthalpy data sets of each sulfide groups in different mesophases in Table 2 were evaluated for correlation through the linear regression method. The correlation coefficients for any groups of solutes in Table 4 exhibit poor correlations between the measured enthalpy data and a single physical property, for example, the coefficient 0.859 account for Group I solutes in the regression of $-\Delta H_{(\mathrm{g}-\mathrm{K})}$ versus $\log L^{16}$. That means at least one other factor would be involved in the correlation relationship. Therefore, we completed MLR calculations using two descriptors, parts of the coefficients in the last three columns in Table 4 are raised, especially the $\log L^{16}-\sum \beta_{2}^{\mathrm{H}}$ bivariate for Group I solutes and the $\sum \beta_{2}^{\mathrm{H}}-E$ bivariate for Group II solutes. Note that $\sum \beta_{2}^{\mathrm{H}}$ provide a great influence on the chromatographic retention in any mesophases of the synthetic copper complex-containing polymer, which acidity is favorable to the proposed ligandexchange mechanism.

As giving an insight into the Group I solutes, the two linear equations, $-\Delta H_{(\mathrm{g}-\mathrm{K})}=129.23-32.51 \log L^{16}+$ $165.83 \sum \beta_{2}^{\mathrm{H}}\left(\mathrm{kJ} \mathrm{mol}^{-1}\right), \quad \gamma=0.984$ and $-\Delta H_{(\mathrm{g}-\mathrm{L})}=$ $71.95-6.77 \log L^{16}-3.23 \sum \beta_{2}^{\mathrm{H}}\left(\mathrm{kJ} \mathrm{mol}^{-1}\right), \quad \gamma=0.990$, are compared with the system constants of the $\mathrm{K}$ and $\mathrm{D}_{\mathrm{L}}$ mesophases. They indicate $\sum \beta_{2}^{\mathrm{H}}$ is more contributed to the enthalpy loss than $\log L^{16}$, because the prefixed value of $\sum \beta_{2}^{\mathrm{H}}$ is larger than the one of $\log L^{16}$ (i.e. system constants $165.83>-32.51$ and $-3.23>-6.77)$. Furthermore, if the first equation was subtracted by the second equation, the resultant equation, $\Delta H_{(\mathrm{g}-\mathrm{L})}-\Delta H_{(\mathrm{g}-\mathrm{K})}=\Delta H_{(\mathrm{K}-\mathrm{L})}=$ $57.28-25.74 \log L^{16}+169.06 \sum \beta_{2}^{\mathrm{H}}$, would match the premier findings in 3.1 for the behaviors of di- $t$-butylsulfide and $t$-butyl methylsulfide. Namely, di-t-butylsulfide with the largest $\log L^{16}$ dissolves easily in $\mathrm{D}_{\mathrm{L}}$ and $t$-butyl methylsulfide with the largest $\sum \beta_{2}^{\mathrm{H}}$ in $\mathrm{K}$.

About Group II solutes, the two linear equations, $-\Delta H_{(\mathrm{g}-\mathrm{L})}=-14.31+30.99 E+12.12 \sum \beta_{2}^{\mathrm{H}}\left(\mathrm{kJ} \mathrm{mol}^{-1}\right)$, $\gamma=0.999 \quad$ and $\quad-\Delta H_{(\mathrm{g}-\mathrm{ho})}=-2.63+27.65 E+$ $10.26 \sum \beta_{2}^{\mathrm{H}}\left(\mathrm{kJ} \mathrm{mol}^{-1}\right), \quad \gamma=0.926$, both show the $E$ descriptor dominates over $\sum \beta_{2}^{\mathrm{H}}$ in either $\mathrm{D}_{\mathrm{L}}(30.99>12.12)$ or $\mathrm{D}_{\text {ho }}(27.65>10.26)$. These should denote the great tendency of the mesophases to interact with Group II sulfides through the two unpaired electrons of sulfur atoms. The molecular dimensions or shapes would affect the strength of the polarizability interaction. Besides, the linearity of the second equation, $\gamma=0.926$, is a little far from the ideal. This deviation may be concerned with the kinetics involved in the GC retention, which only depends on the assumption of a pure partitioning process, and/or may be corrected by other descriptors.

\subsection{Domination of mesomorphic structures}

The morphology of stationary structure effectively dominates the selectivity of solutes. The three phases derived from the synthetic side-chained polymer coating on the capillary

Table 4

Correlation coefficients of $-\Delta H^{\mathrm{a}}$ with physical variables ${ }^{\mathrm{b}}$ for dialkylsulfides

\begin{tabular}{|c|c|c|c|c|c|c|}
\hline \multirow[t]{2}{*}{ Descriptors in mesophases } & \multicolumn{3}{|c|}{ Linear regression of $-\Delta H$ with univariate ${ }^{\mathrm{c}}$} & \multicolumn{3}{|c|}{ Multi-linear regression of $-\Delta H$ with bivariate ${ }^{\mathrm{d}}$} \\
\hline & $\log L^{16}$ & $\sum \beta_{2}^{\mathrm{H}}$ & $E$ & $\log L^{16}, \sum \beta_{2}^{\mathrm{H}}$ & $\log L^{16}, E$ & $\sum \beta_{2}^{\mathrm{H}}, E$ \\
\hline \multicolumn{7}{|l|}{ Group I } \\
\hline $\mathrm{K}$ crystalline $\left(30-40^{\circ} \mathrm{C}\right)$ & 0.859 & 0.208 & 0.266 & 0.984 & 0.867 & 0.305 \\
\hline $\mathrm{D}_{\mathrm{L}}$ mesophase $\left(45-70^{\circ} \mathrm{C}\right)$ & 0.989 & 0.331 & 0.282 & 0.990 & 0.996 & 0.498 \\
\hline \multicolumn{7}{|l|}{ Group II } \\
\hline $\mathrm{D}_{\mathrm{L}}$ mesophase $\left(60-75^{\circ} \mathrm{C}\right)$ & 0.164 & 0.856 & 0.168 & 0.991 & 0.168 & 0.999 \\
\hline $\mathrm{D}_{\text {ho }}$ mesophase $\left(80-95^{\circ} \mathrm{C}\right)$ & 0.231 & 0.776 & 0.123 & 0.849 & 0.445 & 0.926 \\
\hline
\end{tabular}

a Enthalpy changes of solutes from the ideal gaseous state to mesophases, as the same data in Table 2.

b The symbols of variables, $\log L^{16}, \sum \beta_{2}^{\mathrm{H}}$ and $E$, represent the same as in Table 1 .

c Correlation coefficients obtained from the linear regression of $-\Delta H$ with the indicated variable.

d Correlation coefficients obtained from the multi-linear regression of $-\Delta H$ with the two indicated variables. 

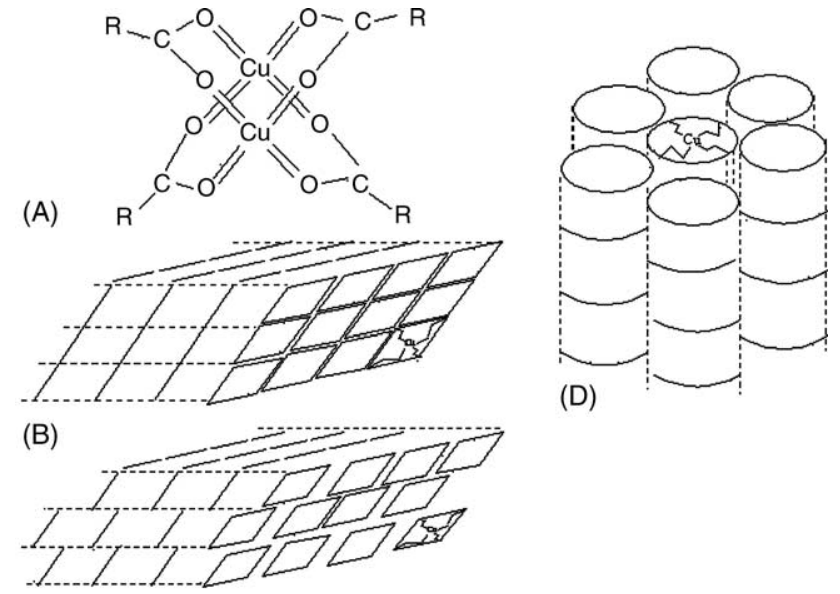

(D)

(C)

Fig. 1. Schematic representation of the stack unit structure (A) and three discotic phases: (B) $\mathrm{K}$, lamellar crystalline, (C) $\mathrm{D}_{\mathrm{L}}$, discotic lamellar, (D) $\mathrm{D}_{\text {ho }}$, discotic hexagonal.

wall showed themselves characteristics after the approval of the multi-linear model.

Every phase consists of discotic units presented in Fig. 1A, which include two copper ions and four long alkyl carboxylates. The phase with higher crystallinity or ordering displays less mobility and more dispersive ability. Here, the general dispersion interactions partly respond to the whole partitioning distribution, although the acid-base interactions do more as the coordination center atoms, $\mathrm{Cu}$ (II), sustain the acidity. These copper atoms are more "visible" and easily approached by donating solutes in the lamellar crystalline $(\mathrm{K})$ phase with the highest crystallinity than in the lamellar discotic $\left(D_{L}\right)$ phase. These two phases are illustrated in Fig. 1B and C.

The discotic hexagonal $\left(\mathrm{D}_{\text {ho }}\right)$ phase shown in Fig. $1 \mathrm{D}$ is less dispersive with less ordering between columns than the $\mathrm{K}$ and $\mathrm{D}_{\mathrm{L}}$ phases. The dipolarity or polarizability contributions from unpaired electrons of sulfur atoms are thus enhanced in the moderately polarizable $\mathrm{D}_{\text {ho }}$ phase and even stronger than the acid-base interactions. In addition, the looser bondage between columns apparently further allows the alkyl substituents of sulfide solutes to enter into the spaces between the disk-like stacks in a column. The substituents, such as disec-butyl and pentamethylene groups, with disk-like shape are more easily inserted into the slot entrances.

\section{Conclusions}

To evaluate the thermodynamic contributions to the retention of dialkylsulfides on the synthesized metallomesogenic polymer by gas chromatography, the measurement of thermodynamic parameters through plotting $\ln \left(t_{\mathrm{r}}-t_{\mathrm{o}}\right)$ versus $T^{-1}$ and $\ln \alpha$ versus $T^{-1}$ accomplishes the descriptive statement about the chromatographic mechanisms and the assess- ment of selectivity. Under the multi-linear model, we suggest four linear equations to describe the quantitative interactions between the sulfide probes and mesophases. The acid-base interaction prevails in the lamellar crystalline $(\mathrm{K})$ phase and the polarizability interaction in the discotic hexagonal $\left(D_{h o}\right)$ phase. The dispersion interaction is easily found in the phases with higher crystallinity. The discotic lamellar $\left(\mathrm{D}_{\mathrm{L}}\right)$ phase with gentle ordering will perhaps experience all these interactions. This work will not only approve the well separation of dialkylsulfides by our GC system, but also benefit the researches in the mesomorphic structure design for the analysis of certain analytes.

\section{References}

[1] J.L. Serrano (Ed.), Metallomesogens-synthesis, properties, and applications, VCH, Weinheim, 1996.

[2] J. Barbera, A.M. Levelut, M. Marcos, P. Romero, J.L. Serrano, Liq. Cryst. 10 (1991) 119.

[3] S.A. Hudson, P.M. Maitlis, Chem. Rev. 93 (1993) 861.

[4] J. Barbera, E. Cavero, M. Lehmann, J.L. Serrano, T. Sierra, J.T. Vazquez, J. Am. Chem. Soc. 125 (2003) 4527.

[5] J. Barbera, R. Iglesias, J.L. Serrano, T. Sierra, M.R. de la Fuente, B. Palacios, M.A. Perez-Jubindo, J.T. Vazquez, J. Am. Chem. Soc. 120 (1998) 2908

[6] C.C. Hu, C.Y. Liu, Anal. Chim. Acta 332 (1996) 23.

[7] C.T. Chou, Y.F. Pai, C.C. Lin, T.K. Misra, C.Y. Liu, J. Chromatogr. A 1043 (2004) 255.

[8] C.Y. Liu, C.C. Hu, J.L. Chen, K.T. Liu, Anal. Chim. Acta 384 (1999) 51.

[9] C.Y. Liu, C.C. Hu, C.L. Yang, J. Chromatogr. A 773 (1997) 199.

[10] C.Y. Liu, J.L. Chen, C.C. Shiue, K.T. Liu, J. Chromatogr. A 862 (1999) 65.

[11] C.Y. Liu, S.H. Yang, M.H. Chau, C.C. Shiue, J. Chromatogr. A 933 (2001) 117.

[12] L. Rohrschneider, Chromatographia 37 (1993) 250.

[13] L. Rohrschneider, Chromatographia 38 (1994) 679.

[14] T.O. Kollie, C.F. Poole, J. Chromatogr. 556 (1991) 457.

[15] K.E. Miller, T.J. Bruno, J. Chromatogr. A 975 (2002) 311.

[16] T.J. Betts, J. Chromatogr. 588 (1991) 231.

[17] K. Héberger, M. Görgényi, J. Chromatogr. Sci. 39 (2001) 113 120.

[18] D.E. Martire, A. Nikolic, K.L. Vasanth, J. Chromatogr. 178 (1979) 401.

[19] E.F. Meyer, J. Chem. Educ. 50 (1973) 191.

[20] S. Ghodbane, G.A. Oweimreen, D.E. Martire, J. Chromatogr. 556 (1991) 317.

[21] M.H. Abraham, Chem. Soc. Rev. 22 (1993) 73.

[22] M.H. Abraham, H.S. Chadha, in: V. Liska, B. Testa, H. van de Waterbeemed (Eds.), Lipophilicity in Drug Action and Toxicology, VCH, Weinheim, 1996, p. 311.

[23] M.H. Abraham, C.F. Poole, S.K. Poole, J. Chromatogr. 842 (1999) 79.

[24] C.F. Poole, S.K. Poole, J. Chromatogr. 965 (2002) 263.

[25] J.A. Platts, M.H. Abraham, Environ. Sci. Technol. 34 (2000) 318.

[26] M.H. Abraham, M.R. Gola, R. Kumarsingh, J.E. Cometto-Muniz, W.S. Cain, J. Chromatogr. B 745 (2000) 103.

[27] M.H. Abraham, Chem. Soc. Rev. 22 (1993) 73.

[28] J.A. Platts, D. Butina, M.H. Abraham, A. Hersey, J. Chem. Inf. Comp. Sci. 39 (1999) 835. 